\title{
Depressive Psychosis Post Total Laryngectomy: A Case Report and Literature Survey
}

\author{
Authors \\ Akpalaba I.O.*, Emokpaire E. \\ Department of Ear, Nose and Throat, University of Benin Teaching Hospital, \\ Benin City, Edo State. \\ Corresponding Author
}

Akpalaba I.O.

Email: drogeakpalaba@gmail.com,+2348035670178

\begin{abstract}
The aim is to emphasize the clinical challenges associated with patient's relative interference with clinical management. This is the first report in the department of a total laryngectomee developing depressive psychosis.

A 70 year-old male with 2years history of unremitting hoarseness and difficulty in breathing of 2 months duration. He took alcohol for 34years, did not smoke, was not a known hypertensive but had elevated blood pressure. He was stridulous. Laryngoscopy showed warty supraglottic mass. Assessment of upper airway obstruction secondary to laryngeal tumour was made. He had Emergency tracheostomy, Direct Laryngoscopy (DL) and Biopsy. Findings were normal trachea, warty growth from glottic to supra-glottic region. Histology was well differentiated invasive squamous cell carcinoma. Computed Tomographic (CT) Scan of Larynx was normal. He declined Total Laryngectomy. DL and biopsy was repeated and histology was severe laryngeal dysplasia. Patient and relatives doubted the result. DL and Biopsy was redone. Histology reported well differentiated keratinizing squamous cell carcinoma. A repeat CT Scan of Larynx showed laryngeal tumour. He had total laryngectomy. Findings were Transglottic fleshy tumour invading the tracheal rings. He developed pharyngocutaneous fistula, managed conservatively. He exhibited abnormal stereotyped movements, insomnia, refusal of meals, aggressiveness and violent tendencies. Mental health team placed him on Haloperidol, then Fluoxetine and frequent visitations. He had series of depressive symptoms, which improved on Risperidone.

Depressive psychosis in a laryngectomee is a major medical concern which needs appropriate management to improve patient's quality of life.
\end{abstract}

Keywords: Total laryngectomy, Depression, Psychosis.

\section{Introduction}

The larynx is the voice box. It has 3 sub-sites, the supraglottis which extends from the epiglottis superiorly to the true vocal folds inferiorly, the glottis which are the true vocal cords and the subglottis which extends from the true vocal folds superiorly to the cricoid cartilage inferiorly.
Removal of the larynx is called laryngectomy. ${ }^{1}$ Total laryngectomy is the removal of the entire larynx while partial laryngectomy involves removal of part of the larynx. Total laryngectomy is indicated in advanced cancer of the larynx. Anyone who has undergone total laryngectomy is called a Laryngectomee. ${ }^{1}$ The key to the 
management of a laryngectomee is multidisciplinary approach involving Otolaryngologist, speech and language pathologist/therapist, dietician, medical psychologist, trained ENT Nurse, Radio-Oncologist, Pathologist, Chest physician, Cardiologist, Physiotherapist and Occupational therapist.
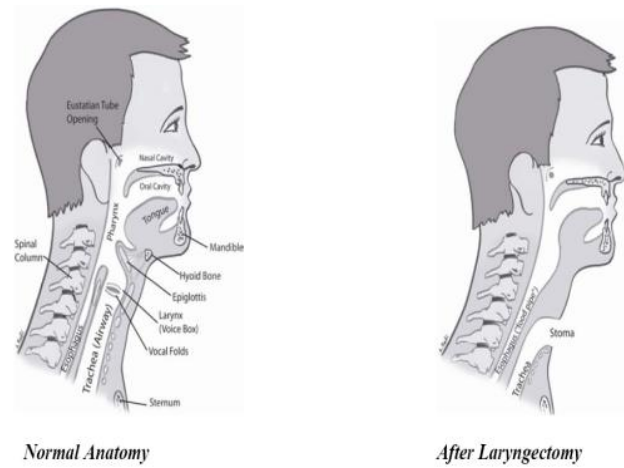

Figure 1: Anatomy of larynx pre- and Post Total Laryngectomy ${ }^{1}$

\section{Statement of problem}

A laryngectomee is posed with multiple life threatening challenges which include permanent loss of voice, permanent tracheostomy, pharyngocutaneous fistula, difficulty with swallowing, recurrent chest infection, loss of valsava manoeuvre causing inability to bear weight or cough effectively and inability to achieve spontaneous vertex delivery in females. ${ }^{1}$ A tracheostomy is an alternate airway created by making a hole in the trachea through the anterior neck skin and maintained with a tube called tracheostomy tube. ${ }^{2}$ This hole through the skin, medically called a stoma, is prone to infections. The overall consequence of these challenges is depression and psychosis leading to poor quality of life.

One of the options to avert permanent voice loss includes training a laryngectomee to achieve oesophageal voice. ${ }^{1} \quad$ Tracheo-oesophageal puncture or fistula, a surgical procedure made during or after total laryngectomy through which voice prosthesis is inserted and electronic larynx, an electronic device which amplifies voice, help in voice rehabilitation. ${ }^{1}$ However the voice breaks.

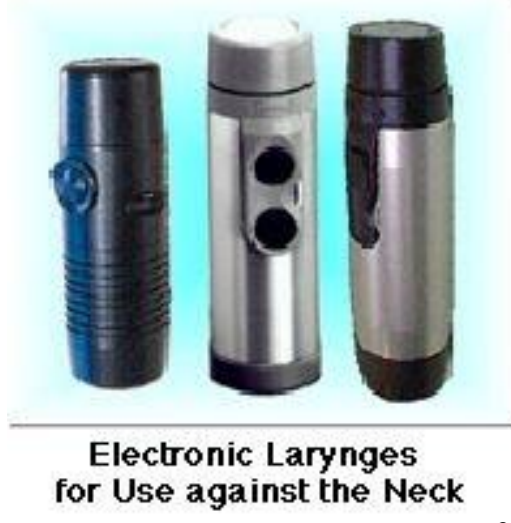

Figure 2: Electronic Larynx ${ }^{3}$

\section{Literature Survey}

Depressive Psychosis is a subtype of major depression that occurs when a severe depressive illness includes some form of psychosis. ${ }^{4}$ The psychosis could be hallucinations (such as hearing a voice telling you that you are not good or worthless), delusions (such as, intense feelings of worthlessness, failure or having committed a sin) or some other break with reality. Depression occurs when you have negative feelings that affect your daily life. These feelings include sadness, hopelessness, guilt, irritability, changes in eating and sleeping. ${ }^{4}$

Depressive psychosis does not have a known cause. According to the journal BMC Psychiatry, older adults are at greatest risk for depressive psychosis. ${ }^{5}$ A combination of antidepressants and antipsychotics are used for the treatment. Examples of the antidepressants include selective serotonin reuptake inhibitors (SSRIs), such as fluoxetine (Prozac). ${ }^{4,5}$ This may be combined with an atypical antipsychotic, such as olanzapine (Zyprexa), quetiapine (Seroquel), risperidone (Risperdal). ${ }^{4}$ Psychotherapy is also used to manage symptoms and overcome fears. A high rate of depression (29\%) was demonstrated in laryngectomees with olfactory dysfunction. ${ }^{6} \mathrm{~A}$ study on non-surgical patients with olfactory impairment suggested that overall life satisfaction was positively correlated with smell scores. ${ }^{7}$ Reasons for this correlation include significant impairments in the areas of safety, personal hygiene, eating, and social interaction. ${ }^{7}$ Personal safety can be impacted as patients are unable to 
use environmental clues to warn themselves of potentially dangerous situations (e.g., detection of smoke, leaking gas, spoiled food). ${ }^{7}$ Poor perception of personal odours can also influence the ability to form and maintain social relationships. ${ }^{8}$ Sense of smell and memory are closely linked with certain odours triggering memories from the past to surface. ${ }^{8}$ This link is severed in those individuals with impaired smell. As flavor perception has input from olfaction, the perception of taste may also be negatively impacted. Assessment of dietary behavior in 176 non-surgical individuals with olfactory dysfunction showed that $29 \%$ of them ate less following the onset of loss of smell, $31 \%$ ate less in between meals, $35 \%$ to $49 \%$ had less social interaction during mealtimes. ${ }^{6}$ In a cohort study, it was found that $89 \%$ of the patients had postoperative reduction in olfaction while 63\% reported changes in taste. ${ }^{9}$ These studies reemphasize the need to heighten attention on potential changes in olfaction and gustation post total laryngectomy.

\section{Method}

A case study of a 70 year old man who presented in March 2010 with a 2 year history of hoarseness and 2 months duration of progressively worsening difficulty in breathing. There was associated, hawking, dyspnoea on mild exertion and cough productive of white sputum. No history of mental illness in the family. No previous history of mental ill health. He was not a known hypertensive or diabetic, but had elevated blood pressure. He took alcohol for 34 years. He was conscious and alert but stridulous in the inspiratory phase.

He had a flexible fibreoptic laryngoscopy which showed pooling of saliva in the piriform fossae and warty supraglottic mass. Diagnosis of upper airway obstruction secondary to laryngeal tumour was made, for which he had an emergency tracheostomy, rigid direct laryngoscopy and biopsy. Findings were normal trachea, warty growth friable and non hemorrhagic jutting from glottic to supraglottic region. The histology was well differentiated invasive squamous cell carcinoma. Computed tomographic scan of the larynx done thereafter showed normal laryngeal air column with a polypoidal mass on the left cord. Relatives, among who were medical personnel, doubted the result; convinced the patient and he declined total laryngectomy. Four months after, a repeat direct laryngoscopy and biopsy done showed similar findings. The histology was severe laryngeal dysplasia which was doubted by the managing team. Direct laryngoscopy and biopsy was repeated the $3^{\text {rd }}$ time three months after the last procedure. The histology reported well differentiated keratinizing squamous cell carcinoma. A repeat computed tomographic scan of the larynx showed laryngeal tumour with normal adjacent laryngeal cartilages. He then had a total laryngectomy 3 months following last surgical procedure. Findings were transglottic fleshy tumour invading the tracheal rings, $\mathrm{T}_{4} \mathrm{a} \mathrm{N}_{0} \mathrm{M}_{0}$ (Cancer Stage 1VA). ${ }^{10}$ On the $6^{\text {th }}$ day post operative he developed stoma wound infection which progressed to a pharyngocutaneous fistula. This was managed conservatively. He was on serial fasting and random blood sugar monitoring which was within the normal range.

On the $29^{\text {th }}$ day post operatively he exhibited abnormal stereotyped movements, insomnia, refusal of meals, aggressiveness and violent tendencies. Mental health team reviewed and placed him on Haloperidol. Following commencement of haloperidol he was always weeping, not obeying instructions, frequently drowsy with serial hypokalemia. Haloperidol was withdrawn and replaced with Fluoxetine. Frequent visitation from his children was encouraged. Despite these, he had series of acute exacerbation of depressive and psychotic symptoms which then improved on Risperidone. Blood pressure control was achieved, pharyngocutaneous fistula healed and feeding improved per orale.

He was then discharged for chemo- irradiation, which he completed and has been on regular ear nose and throat clinic visits. 
On his $11^{\text {th }}$ clinic visit post irradiation, the fasting blood sugar was elevated $(217 \mathrm{mg} / \mathrm{dl})$ for which he was admitted and managed by the endocrinologist as a case of type 2 diabetic mellitus. He was discharged home after achieving good glycaemic control. He is now on regular clinic visits and has made 22 visits post irradiation. No recurrence of depressive or psychotic symptoms. He is now 8 years survival post total laryngectomy.

\section{Discussion}

\section{Diagnostic tools}

In surgical cases, diagnosis is based on triple diagnostic tools namely: history taking, clinical diagnosis from examination and radiological or laboratory diagnosis. When two of the diagnostic tools are positive, treatment or management line mapped out is implemented.

This patient had classical symptoms and signs of upper airway obstruction secondary to laryngeal tumour which was further confirmed by direct laryngoscopy and biopsy. This agreed with the principle of triple diagnosis, but due to discrepancy between the first radiologic investigation (CT scan of larynx), and the histological result, the relatives interfered and patient therefore declined surgery.

This adversely affected the patient's management and outcome.

\section{Prolonged hospital stay}

A laryngectomee stays a maximum of 14 days on admission when there is no associated postoperative complication. This patient developed pharyngocutaneous fistula postoperatively. This is a major complication of total laryngectomy arising most times due to insufficient mucosal tissue for pharyngeal repair during the surgery. The delay in commencement of treatment due to relative's interference most likely caused progression of the disease. The advanced laryngeal cancer which involved the tracheal rings contributed to the development of pharyngocutaneous fistula and subsequent prolonged stay on admission. Prolonged hospital stay could cause depressive episodes.

\section{Risperidone and Type 2 Diabetic Mellitus ${ }^{4}$}

Risperidone is a second generation atypical antipsychotic drug with low incident of extra pyramidal side effect. Its major side effect is type 2 diabetic mellitus. ${ }^{4}$ This patient most likely developed type 2 diabetic mellitus from Risperidone.

\section{Post laryngectomy challenges and coping with reality}

The diagnosis of cancer and its chronicity is most times a difficult task. Most patients live in doubt, this alone causes major depression.

This patient is a lover of native meals. It was difficult for him to accept the reality of Nasogastric tube (NG) feeding post operative till when he can tolerate orally. Physical impairment and doubting the inevitable could cause depressive psychosis.

\section{Loneliness and abandonment by loved ones}

Psychotherapy plays a great role in cancer management. This patient's relatives were not always around due to distance and busy schedule. His condition improved when there was frequent visitation from his wife and children.

\section{Conclusion}

Total laryngectomy is accompanied by life threatening challenges including depressive psychosis which is a major medical concern. Hence, multidisciplinary approach is the key to the management of a laryngectomee to improve the patient's quality of life.

\section{Future Scope}

We therefore recommend reinforcement of preoperative counselling on potential changes post total laryngectomy and medical psychotherapy in the management of cancer patients. 


\section{References}

1. Sievers, Walker, Raf 11. Laryngectomy. UCDavis Health System, 2007: 6- 20. Ucdavis.edu. Assessed on 07/04/2016

2. Hilgers FJ, van Dam FS, Keyzers S, Koster MN, van As CJ, Muller MJ. Rehabilitation of olfaction after laryngectomy by means of a nasal airflowinducing maneuver: The "polite yawning" technique. Arch Otolaryngol Head Neck Surg 2000;126:726-32.

3. Ward E, Rumbach A, van As-Brooks CJ. Olfaction following total laryngectomy. J Laryngol Voice 2012;2:10-20

4. Proietto J, Dunlop E.Diabetes and Antipsychotic Drugs. Australian prescriber,2004; 27(5):118-119

5. Flint AJ, Meyers BS, Rothschild AJ, Whyte EM, Mulsant BH, Rudorfer MV, Marino P. Sustaining remission of psychotic depression: rationale, design and methodology of STOP PDII. BioMed Central Psychiatry, 2013: 13:38

6. Deems DA, Doty RL, Settle G, MooreGillon V, Shaman P, Mester AF, et al. Smell and taste disorders: A study of 750 patients from the University of Pennsylvania Smell and Taste Center. Arch Otolaryngol Head Neck Surg 1991;117:519-28

7. Miwa T, Furukawa M, Tsukatani T, Costanzo RM, DiNardoLJ, Reiter ER. Impact of olfactory impairment on quality of life and disability. Arch Otolaryngol Head Neck Surg 2001;127:497-503.

8. Aschenbrenner K, Hummel C, Teszmer K, Krone F, Ishimaru T, Seo HS, et al. The influence of olfactory loss on dietary behaviours. Laryngoscope 2008;118:13544.

9. Lennie TA, Christman SK, Jadack RA. Educational needs and altered eating habits following a total laryngectomy. OncolNurs Forum 2001;28: 667-74
10. Larynx. AJCC Cancer Staging Manual. $7^{\text {th }}$ edition, New York, NY: Springer; 2010. 57-62

\section{Author Profile}

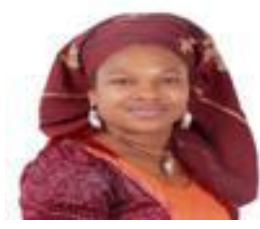

Akpalaba Immaculata Ogechi is a medical doctor, a trained Ear, Nose and Throat specialist (Otorhinolaryngologist) who was among the best ten graduating students of her school, Ebonyi State University Abakaliki, in the final MBBS examination, April 2004. She was the prize winner of the Otorhinolaryngological Society of Nigeria, ORLSON, for the Best Young Consultant Paper Presentation, November, 2015 ORLSON Conference in Calabar, Nigeria. She recently concluded her residency training at the University of Benin Teaching Hospital, UBTH, Benin City, Nigeria. She has interest in Rehabilitative Medicine, facial plastics in Otorhinolaryngology, leadership in health management and entrepreneurship.

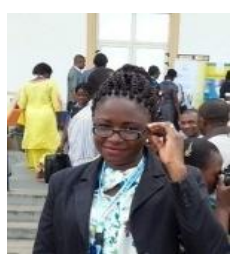

Dr Onohinosen Ehiaghe Emokpaire is a lecturer with the department of surgery, school of medicine. She is an honorary consultant with the University of Benin Teaching Hospital Benin City. She is a fellow of the West African College Of Surgeons and member of the Otorhinolaryngology Society Of Nigeria. She was awarded the best candidate in the Part 1 fellowship examination in otorhinolaryngology by the national post graduate medical college. She has interest in paediatric otolaryngology and general otolaryngology. 